\title{
Inspection Tool for Testing an Electron Beam in an Electromagnetic Lens System
}

\author{
Cheolsu Han ${ }^{1}$, Jong-Man Jeong ${ }^{1}$, Sang-Chul Lee and Jin-Gyu Kim ${ }^{2}$
}

${ }^{1}$ Electron Microscopy Research Center, Korea Basic Science Institute, Daejeon, Republic of Korea.

2. Division of Environmental and Material Sciences, Korea Basic Science Institute, Daejeon, Republic of Korea.

Electron microscopes (EM) such as a scanning electron microscope and a transmission electron microscope (TEM) are powerful tools in nanometer science and technology [1]. Especially in the semiconductor industry, the EMs are widely used to measure a critical dimension of nano-patterns and inspect defects in patterned surface of devices.

The EM uses accelerated electron beam to illuminate or transmit a specimen and obtain an enlarged image of surface or inside structure of that [2]. The accelerated electron beam can be illuminated the specimen by electromagnetic lenses and coils. The electromagnetic lens is a device for the focusing or deflection of moving accelerated electron beam by use of the magnetic force. The electromagnetic lens consists of a coil of copper wires inside the iron pole pieces. We can control the focal length of electromagnetic lens and the deflection angle of the moving accelerated electron beam by controlling a current through the coils [3-4].

There are many condenser lenses and deflection coils in the TEM. The electron beam is illuminated the specimen by passing through the narrow tube of many lenses and coils. The transmitted beam is also illuminated the phosphor screen of the TEM by passing through the narrow tube of some lenses and coils. In order to obtain the TEM image on the screen, the electron beam must be in the optical axis. Using deflection coils, we can control the moving direction of electron beam to the optical axis. However, if the electron beam misaligned to the optical axis, we cannot observe the TEM image.

In this presentation, we introduce an inspection tool for testing and aligning an electron beam in an electromagnetic lens system (see Fig. 1 and 2).

The inspection tool consists of an electron beam tracking and measuring system. The electron beam tracking system includes a small phosphor screen, two cameras and 4-DOF (degree of freedom, X-Y-ZTilt) stage. The electron beam is can be converted a visualized beam by the small phosphor screen. The visualized beam image is can be captured by using the camera. We can determine the current position of the electron beam using the captured image from the camera. The electron beam can be move to the optical axis by controlling the deflection coils. There are a Faraday cage and a pico-ammeter in the electron beam measuring system. The Faraday cage collects controlled electrons by the coils or the lenses. We measured the electron beam current using the pico-ammeter and the Faraday cage [5].

\section{References:}

[1] E. Munro in "Handbook of Charged Particle optics," $1^{\text {st }}$ ed. (CRC press, NY, 1997), Ch. I.

[2] David B. Williams et al, in "Transmission Electron Microscopy: A Textbook for Materials Science," (Springer, 2009), Ch. 9.

[3] C. Han et al, Rev.Sci.Instrum. 88 (2017), p. 023302. 
[4] John T. L. Thong in "ELECTRON BEAM TESTING TECHNOLOGY," (Plenum Press, NY, 1993). [5] The authors acknowledge funding from Korea Basic Science Institute, Grant Number D36612.

(a)

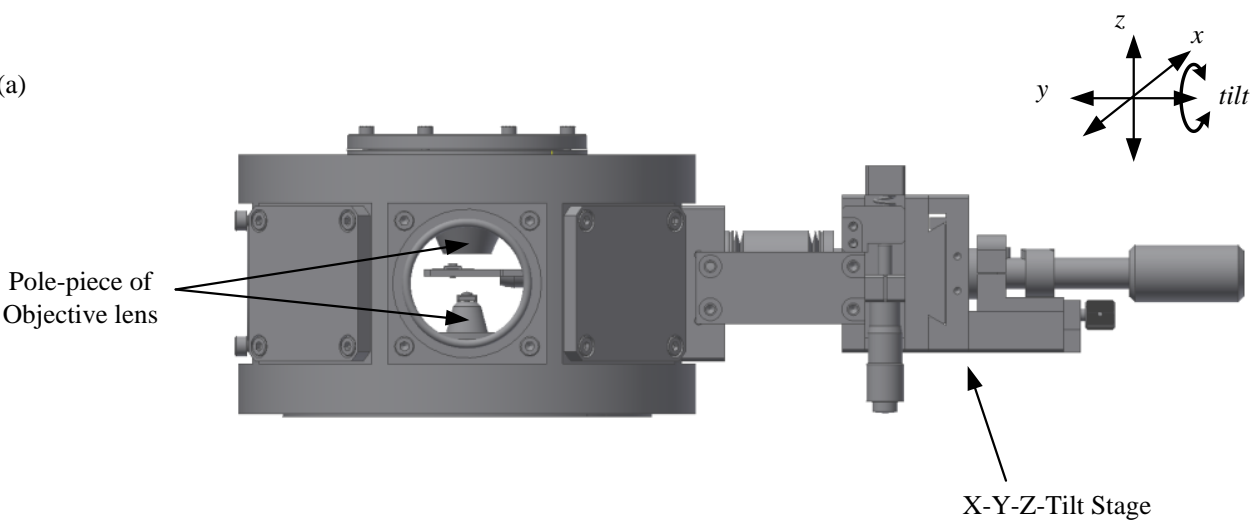

(b)

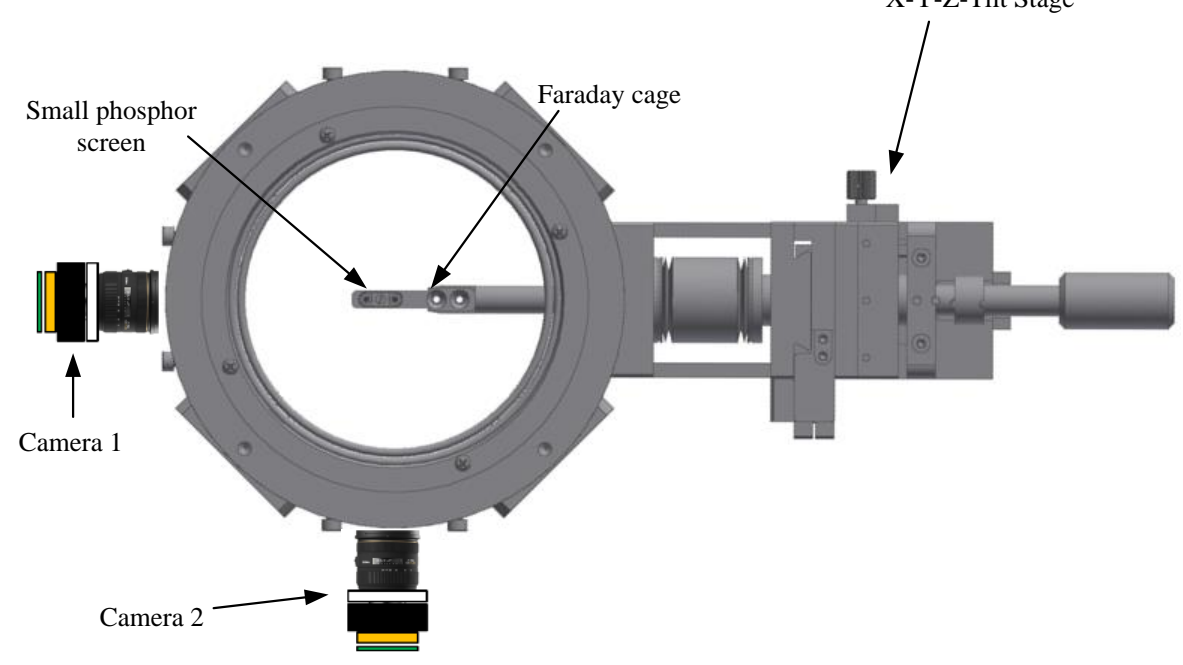

Figure 1. Schematic diagram of the inspection tool (a) side view (b) top view

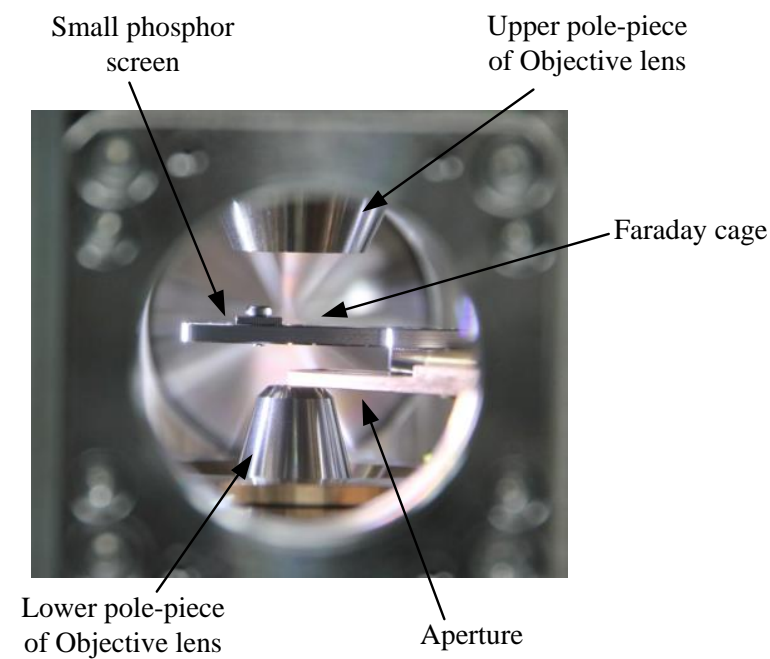

Figure 2. Photograph of the inside of chamber. 